\title{
Synthesis, Thermal and Electrical Properties of 2, 2'-HBBF Copolymer Resin
}

\author{
SANJIOKUMAR S. RAHANGDALE and WASUDEO B. GURNULE*
}

Department of Chemistry, Jagat Arts, Commerce and Indiraben Hariharbhai Patel Science College, Goregaon - 441 801, Maharashtra, India

*Department of Chemistry, Kamla Nehru Mahavidyalaya, Sakkardara Square, Nagpur - 440 009, Maharashtra, India

wbgurnule@yahoo.co.in

Received 12 July 2012 / Accepted 16 August 2012

\begin{abstract}
The copolymer (2, 2'-HBBF) synthesized by the condensation of 2, 2'-dihydroxybiphenyl $\left(2,2^{\prime}-\mathrm{HB}\right)$ and biuret (B) with formaldehyde (F) in the presence of acid catalyst using 1:1:2 molar proportions of the reacting monomers. The thermogravimetric analysis of copolymer resin prepared in the present study has been carried out by non-isothermal thermogravimetric analysis technique in which sample is subjected to condition of continuous increase in temperature at linear rate. The advantage of Freeman-Carroll method is to calculate both the order of reaction (n) and energy of activation in one single stage by keeping heating rate constant. By using data of thermogravimetric analysis, various thermogravimetric parameters like frequency factor $(Z)$, entropy change $(\Delta S)$, free energy change $(\Delta \mathrm{F})$ and apparent entropy $\left(\mathrm{S}^{*}\right)$ have been determined using Freeman-Carroll method. The electrical properties of 2, 2'-HBBF copolymers were measured over a wide range of temperature (303-423 K). From the electrical conductivity of these copolymers, activation energies of electrical conduction have been evaluated and values lies in the range $3.01 \times 10^{-20}-6.43 \times 10^{20} \mathrm{~J} / \mathrm{K}$. On the basis of above studies, these copolymers can be ranked as semiconductors.
\end{abstract}

Keywords: Resin, Synthesis, Electrical conductivity, Thermal degradation

\section{Introduction}

Copolymer resins having good thermal stability have enhanced the development of polymeric materials. The thermal degradation study of copolymers has become a subject of interest. Study of thermal behaviour of copolymers in air at different temperature provides information about the nature of species produced at various temperatures due to degradation. In this laboratory, extensive work on the thermal degradation of copolymers has been undertaken $^{1-2}$. Jadhao et $a l^{3}$ synthesized the terpolymer resin by the condensation of 2, 2'dihydroxybiphenyl with urea and formaldehyde in the presence of $2 \mathrm{M} \mathrm{HCl}$ as a catalyst. They reported thermal studies of the resins have been carried out to determine their mode of decomposition, activation energy, order of reaction, frequency factor, entropy change, free energy and apparent entropy change. Ukey and coworkers ${ }^{4-6}$ synthesized and studied thermal 
properties of some chelate polymers. The decomposition temperature and the order of reaction have been determined by TGA analysis. Thermal data have been analyzed by Freeman-Carroll and Sharp-Wentworth methods and various kinetic and thermodynamic parameters have also been evaluated. Comparable values of parameters indicates common decomposition reaction mode in all chelate polymers.

Patel and Patel $^{7}$ were studied preparation and thermal degradation of 2-hydroxy-4methoxypropiophenone-urea-formaldehyde copolymers. They observed that copolymers prepared using equimolar proportions of reactants are thermally more stable. Gurnule et. al..$^{8-9}$ have reported semiconducting studies of 8-hydroxyquinoline- melamine/biuret-formaldehyde terpolymer resins. Borole and co-workers ${ }^{10}$ synthesized and characterized the poly (aniline-co-o-anisidine-co-otoluidine) this films in inorganic and organic supporting electrolytes. The influence of inorganic and organic supporting electrolytes on electrochemical, optical properties of terpolymer thin films has been investigated. The films were characterized by conductivity measurements using four-probe technique. Borkar et. al. ${ }^{11-12}$ studied electrical and optical properties of conducting copolymer.

\section{Experimental}

2, 2'-Dihydroxybiphenyl (SRL, Mumbai) and biuret (Merck, India) were purified by rectified spirit, formaldehyde (37\%). Double distilled water was used in all the experiments.

\section{Synthesis of 2, 2'-HBBF copolymer resin}

Copolymer resin (2, 2'-HBBF-I) was prepared by condensing 2, 2'-dihydroxybiphenyl (1.86 g, 0.1 mole), biuret ( $1.3 \mathrm{~g}, 0.1 \mathrm{~mole}$ ) and formaldehyde ( $7.5 \mathrm{~mL}$ of $37 \%, 0.2 \mathrm{~mole}$ ) in the presence of $2 \mathrm{M} \mathrm{HCl}(200 \mathrm{~mL})$ as a catalyst at $118 \pm 2{ }^{0} \mathrm{C}$ in an oil bath for $6 \mathrm{~h}^{13-17}$. The solid product obtained was immediately removed from the flask as soon as the reaction period was over. It was washed with cold water, dried and powdered. The powder was repeatedly washed with hot water to remove unreacted monomers. The air-dried copolymer resin was extracted with petroleum ether and diethyl ether to remove copolymer. It was further purified by dissolving in $8 \% \mathrm{NaOH}$ and then was filtered. The copolymer was then precipitated by drop wise addition of 1:1 (v/v) conc. $\mathrm{HCl} /$ water with constant stirring and filtered. The process was repeated twice. The resulting polymer sample was washed with hot water, methanol and dried in a vacuum at room temperature. The purified copolymer resin was finely ground to pass through a 300 mesh size sieve and kept in a vacuum over silica gel. The yield of the copolymer resin was found to be $82 \%$.
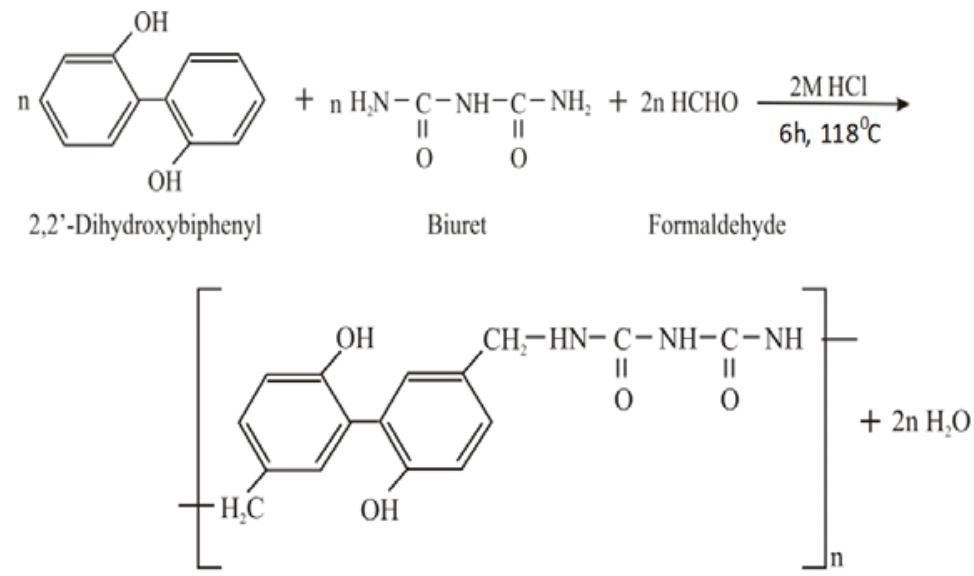

Scheme 1. Proposed reaction for 2,2'-HBBF-1 copolymer resin 
Similarly, the other copolymer resins, 2, 2'-HBBF-II, 2, 2'-HBBF -III and 2, 2'-HBBF -IV were synthesized by varying the molar proportion of the starting monomers i.e. 2, 2'-dihydroxybiphenyl, biuret and formaldehyde in the ratios 2:1:3, 3:1:4 and 4:1:5 respectively.

\section{Characterization}

\section{Instrumentation}

The non-isothermal thermogravimetric analysis was performed in air atmosphere with heating rate of $100{ }^{0} \mathrm{C} \mathrm{min}{ }^{-1}$ using $5-6 \mathrm{mg}$ of samples in platinum crucible from temperature of $400{ }^{\circ} \mathrm{C}$ to $800{ }^{\circ} \mathrm{C}$ and thermograms were recorded for 2, 2'-HBBF sample at SICART, Vallabhvidyanagar, Gujrat. With the help of thermogravimetric data the thermal activation energies $(E a)$ and order of reaction $(n)$ were calculated. Also other thermodynamic parameters such as entropy change $(\Delta S)$, apparent entropy change $\left(S^{*}\right)$ and frequency factor $(z)$ were determined and reported in the Table 1.

\section{Theoretical considerations}

To provide further evidence regarding the degradation system of analyzed compounds, we derived the TG curves by applying an analytical method proposed by Sharp-Wentworth and Freeman- Carroll.

\section{Freeman-Carroll method}

The straight-line equation derived by Freeman and Carroll ${ }^{18}$, which is in the form of $\mathrm{n}$

$$
[\Delta \log (\mathrm{dw} / \mathrm{dt})] / \Delta \log \mathrm{Wr}=(-\mathrm{E} / 2.303 \mathrm{R}) . \Delta(1 / \mathrm{T}) / \Delta \log \mathrm{Wr}+\mathrm{n}
$$

Where, $\mathrm{dw} / \mathrm{dt}=$ rate of change of weight with time. $\mathrm{Wr}=\mathrm{Wc}-\mathrm{W}, \mathrm{Wc}=$ weight loss at completion of reaction. $\mathrm{W}=$ fraction of weight loss at time t. Ea = energy of activation. $\mathrm{n}=$ order of reaction.

The plot between the terms $[\Delta \log (\mathrm{dw} / \mathrm{dt})] / \Delta \log \mathrm{Wr} v s . \Delta(1 / \mathrm{T}) / \Delta \log \mathrm{Wr}$ gives a straight line from which slope we obtained energy of activation (Ea) and intercept on $Y$-axis as order of reaction $(n)$. The change in entropy $(\Delta S)$, frequency factor $(z)$, apparent entropy $\left(S^{*}\right)$ can also be calculated by further calculations.

\section{Sharp -wentworth method}

Using the equation derived by Sharp and Wentworth ${ }^{19}$,

$$
\log [(\mathrm{dc} / \mathrm{dT}) /(1-\mathrm{c})]=\log (\mathrm{A} / \beta)-[\mathrm{Ea} / 2.303 \mathrm{R}] .1 / \mathrm{T}
$$

Where, $d c / d T=$ rate of change of fraction of weight with change in temperature $\beta=$ linear heating rate $d T / d t$.

By plotting the graph between log dc/dt/ (1-c) vs. 1/T we obtained the straight line which give energy of activation ( $E a$ ) from its slope. Where $\beta$ is the conversion at time $t$, $R$ is the gas constant $\left(8.314 \mathrm{Jmol}^{-1} \mathrm{~K}^{-1}\right)$ and $T$ is the absolute temperature. The plots (Figure 1) give the activation energies at different stages of degradation reaction take place. This isoconversional (model-free) kinetic methods use to check the variation of the apparent activation energy values with degree of degradation. This kinetic analysis should be a starting point for obtain the useful information on the behaviour of the sample (Table 1).

\section{Electrical properties}

The resistance of copolymers was measured over a wide range of temperature (303-423 K) in their pellets form using Auto Comput LCR-Q meter 4910. The instrument could read and measure upto $0.1 \mathrm{ohm}$ to $10^{6}$ Megaohm and test voltage varied from 50-500 volts; the accuracy 
being at the lower range $+5 \%$. To prepare the pellets, the copolymer was thoroughly ground with an agate pestle and mortar. The well powdered copolymer was pelletalized isostatically in a steel die at 10 tones/inch ${ }^{2}$ with the help of a hydraulic press. Pellets of $1.29 \mathrm{~cm}$ in diameter and nearly 0.13 to $0.16 \mathrm{~cm}$ thickness were prepared. The pellet of the test sample was put in a typical cell fabricated in this laboratory and resistance in Megaohms was measured as a function of temperature.

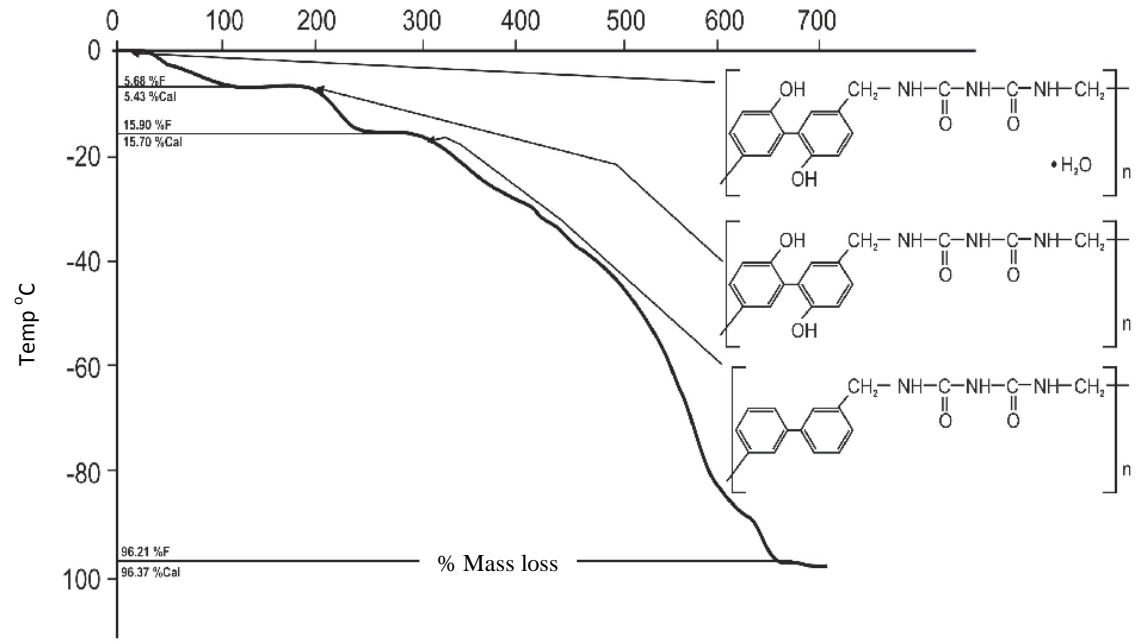

Figure 1. TG plot of 2,2'-HBBF-1 copolymer

Table 1. Kinetic parameters of 2, 2' - HBBF Copolymers

\begin{tabular}{ccccccccc}
\hline & & $\begin{array}{c}\text { Activation } \\
\text { energy, } \\
\text { S.No. }\end{array}$ & Copolymer & $\begin{array}{c}\text { Entropy } \\
\text { Change, } \\
\text { kJ mole }\end{array}$ & $\begin{array}{c}\text { J } \\
\text { Jenee } \\
\text { engy } \\
\text { change, kJ }\end{array}$ & $\begin{array}{c}\text { Frequency } \\
\text { Factor, s }\end{array}$ & $\begin{array}{c}\text { Apparent } \\
\text { entropy } \\
\text { change, kJ }\end{array}$ & $\begin{array}{c}\text { Order of } \\
\text { reaction }\end{array}$ \\
\cline { 3 - 9 } & & FC & SW & $\Delta \mathrm{S}$ & $\Delta \mathrm{F}$ & $\mathrm{Z}$ & $\mathrm{S}$ & $\mathrm{n}$ \\
\hline 1. & 2, 2'-HBBF -I & 19.26 & 19.20 & -8.4468 & 15.54 & 79.7 & -25.30 & 0.94 \\
2. & 2, 2'-HBBF -II & 20.29 & 21.01 & -8.4974 & 16.51 & 79.0 & -25.31 & 0.88 \\
3. & 2, 2'-HBBF -III & 17.11 & 18.02 & -8.7426 & 13.09 & 32.0 & -26.22 & 1.01 \\
4. & 2, 2'-HBBF -IV & 20.56 & 20.81 & -8.6073 & 16.56 & 60.0 & -25.59 & 0.92 \\
\hline
\end{tabular}

$F C=$ Freeman-Carroll Method,$S W=$ Sharp-Wentworth Method

\section{Result and Discussion}

Thermogravimetric analysis

Decomposition pattern of representative system and TGA curve of 2, 2'-HBBF copolymer resins are shown in Figure 1. The kinetic parameters for the thermal degradation have been evaluated from the thermogram by using Freeman-Carroll Method and Sharp-Wentworth Method which are presented in Table 1. Examination of thermogram and TG data of 2, 2'-HBBF copolymer resins showed that these polymers have resistance to elevated temperatures. Thermogram of all 2, 2'-HBBF copolymer exhibited two stages of decomposition after loss of water molecules. It is observed from TG data of these copolymer resins that the sample lost 3.81 to $6.58 \%$ of its weight when the temperature was raised to $180{ }^{\circ} \mathrm{C}$. This initial weight loss may be attributed to solvent or moisture entrapped in the copolymer samples ${ }^{21-24}$. First decomposition step in 2, 2'-HBBF copolymer resin represents 
degradation of both phenolic hydroxyl groups. Second decomposition step in case of all 2, 2'-HBBF copolymer resin shows the complete degradation of aromatic biphenyl nucleus and side chain attached to aromatic biphenyl ring.

The thermal decomposition can be explained tentatively by considering one repeating unit of the copolymer resin. The initial decomposition temperature is frequently used to define the relative thermal stability of copolymer resins. However, the degree of decomposition is neglected. From the decomposition temperature data, it can be concluded that the thermal stability of copolymer resin is in the order: 2, 2'-HBBF-I > 2, 2'-HBBF-II > 2, 2'-HBBF-III > 2, 2'-HBBF-IV.

From the above order it can be concluded that the higher thermal stability of copolymer sample may be due to stronger intermolecular hydrogen bonding present in polymer chain and it also indicates that thermal degradation behavior is regardless of their molecular weight or independent of structure and molar ratio of monomers. It may be due to collinear arrangement of monomer unit in polymer chain, which allow the formation of stronger intermolecular hydrogen bonding ${ }^{21,24}$. From the data, it can be observed that the values of the thermodynamic parameters are nearly the same for each copolymer resin. The similarity of the thermodynamic parameters indicates a common decomposition reaction mode ${ }^{9,22}$. With regards to the abnormally low value of $\mathrm{Z}$, it can be concluded that the reaction of decomposition of copolymers can be classified as slow reaction and no other obvious reason can be given ${ }^{22}$.

The negative values of entropy $(\Delta S)$ indicate that the activated polymer has more ordered structure than the reactants and the reactions are slower than normal. This is further supported by low $\mathrm{Z}$ values ${ }^{21}$. Our observations are further supported by findings of earlier workers ${ }^{22}$. Higher values of activation energy suggest the higher stability ${ }^{24}$. Thus, the thermal activation energy is in the order: 2, 2'-HBBF-IV > 2, 2'-HBBF-II > 2, 2'-HBBF-I > 2, 2'-HBBF-III.

\section{Electrical conductivity measurements}

The DC resistivities of the 2, 2'-HBBF copolymer resins were measured in the temperature range of 303-423 $\mathrm{K}$. The electrical conductivity of copolymer samples at room temperature vary from $7.5 \times 10^{-13}$ to $5.1 \times 10^{-12}$ Siemen $^{25-28}$. The temperature dependence of the electrical conductivity (Figure 2) is found to be linear in the temperature range under study showing thereby that Wilson's exponential law is obeyed.

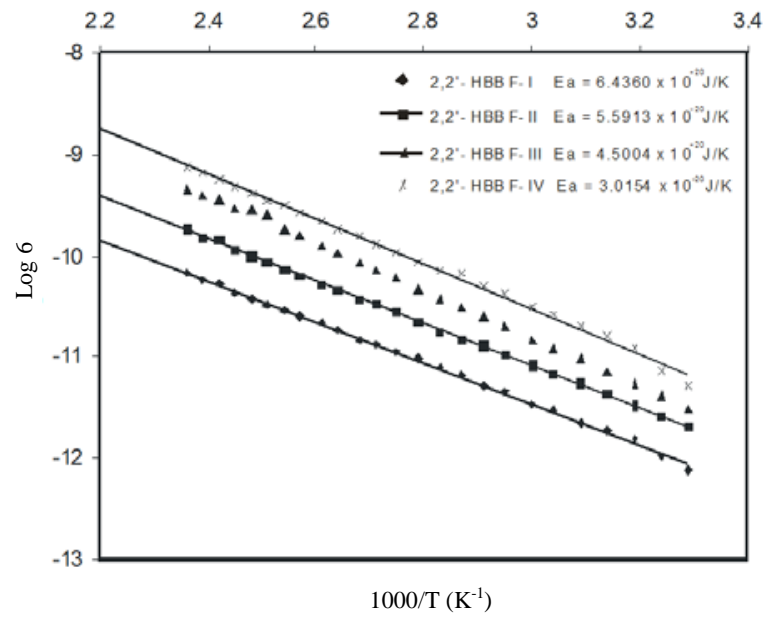

Figure 2. Electrical conductivity plots of 2,2'-HBBF copolymers, temperature dependence of $\log \sigma$ 
Examination of the plots also revealed that the electrical conductivity of the copolymers increases with increase in temperature. Hence, these copolymers may be ranked as semiconductors. The activation energy calculated from the slopes of the plots is found to be in the range of $6.43 \times 10^{-20}$ to $3.01 \times 10^{-20} \mathrm{~J} / \mathrm{K}$. The low magnitude of activation energy may be due to the large number of $\pi$ (pi) electrons. The activation energy was found to decrease in the order, 2, 2'-HBBF-I > 2, 2'-HBBF-II > 2, 2'-HBBF-III > 2, 2'-HBBF-IV. The above decreasing order of activation energy of electrical conduction may be due to introduction of more aromatic skeleton (thereby increase in no of $\pi$ electrons) in the repeat unit structure of the copolymers.

\section{Conclusion}

The 2, 2'-HBBF-I copolymer based on the condensation polymerization of o-aminophenol and urea with formaldehyde in the presence of acid catalyst has been prepared. In TG the energy of activation evaluated from the Freeman-Carroll and Sharp-Wentworth methods are found to be nearly equal and the thermodynamic parameters obtained from Freeman-Carroll method are found to similar, indicating the common reaction mode. Low values of frequency factor $(Z)$ may be concluded that the decomposition reaction of 2, 2'-dihydroxybiphenyl-biuret-formaldehyde copolymer can be classified as 'slow reaction'. Electrical conductivity of each of these copolymer resins increases with increase in temperature. Hence, these copolymers may be ranked as semiconductors.

\section{Acknowledgement}

The authors express their sincere thanks to The Director, Laxminarayan Institute of Technology, Nagpur for cooperation and for providing necessary laboratory facilities. They are also thankful to the UGC for financial support.

\section{References}

1. Tarase M V, Zade A B and Gurnule W B, J Ultra Sci., 2007, 3, 41.

2. $\quad$ Gurnule W B, Juneja H D and Paliwal L J, Orient J Chem., 1999, 15, 283-288.

3. Jadhao M M, Paliwal L J and Bhave N S, J Appl Polym Sci., 2006, 101(1), 227-232.

4. Bonde A D, Ukey V V, Juneja H D, Ghuble R S and Hussain R, Mat Sci Eng B, 2006, 132(1-2), 16-19.

5. Ukey V V, Juneja H D, Borkar S D, Ghuble R S and Naz S, Mat Sci Eng B, 2006, 132(1-2), 34-38.

6. $\quad$ Ukey V V and Juneja H D, J Appl Polym Sci., 2006, 99(1), 273-278.

7. $\quad$ Patel G C and Patel M M, Proc Indian Acad Sci (Chem Sci)., 1991, 103(4), 539-547.

8. $\quad$ Gurnule W B, Juneja H D and Paliwal L J, Asian J Chem., 2000, 12, 753.

9. Gurnule W B, Juneja H D and Paliwal L J, Ultra Sci., 2001, 13, 222.

10. Borole D D, Kapadia U R, Mahulikar P P and Hundiwale D G, J Mater Sci., 2006, 41, 1983.

11. Borkar A D, Umare S S and Gupta M C, Prog Cryst Grow Charact Mat., 2002, 44, 88.

12. Borkar A D, Umare S S and Gupta M C, Polym Plast Tech Eng., 2001, 40(2), 225.

13. Maki T, Ishihara K and Yamamoto H, Tetrahedron, 2007, 10, 1016.

14. Masram D T, Karia K P and Bhave N S, e-Polymers, 2007, 075, 1-11.

15. Patel M M, Kapadia M A, Patel G P and Joshi J D, J Appl Polym Sci., 2007, 106(2), 1307-1317.

16. Patel M M, Patel G C and Pancholi H B, J Indian Chem Soc., 1995, 72, 533-535.

17. Patel S A, Shah B S and Patel R M, Iran Polym J, 2004, 13(6), 445-453. 
18. Freeman E S and Carroll B, J Phys Chem., 1958, 62(4), 394-397.

19. Sharp J B and Wentworth S A, Anal Chem., 1969, 41(14), 2060-2062.

20. Jadhao M, Paliwal L J and Bhave N S, Indian J Chem., 2005, 44-A, 1110-1113.

21. Jacobs P W M and Tompkins F C, Chemistry of the Solid State, ed. W. E. Garner Publication, London, 1955, 188.

22. Lingala P S, Juneja H D and Paliwal L J, Thermans, Gorakhpur, 2000, 245-247.

23. Michael P E P, Lingala P S, Juneja H D and Paliwal L J, J Appl Polym Sci., 2004, 92(4), 2278-2283.

24. Nadia Ahmed Mohamed N A and Abeer Obaid Hamad Al-Dossary A O, Polym Degrad Stab., 2003, 79(1), 61-75.

25. Nandekar K A, Dontulwar J R and Gurnule W B, Der Pharma Chemica, 2012, 4(4) 1644-1652.

26. Gurnule W B and Butoliya S S, J Appl Polym Sci., 2011, 122, 2181-2188.

27. Zhang Q, Jin H, Wang X and Jing X, Synth Metals, 2001, 123(3), 481-485.

28. Ahamed M A R, Azarudeen R S, Mylswamy K and Burkanudeen A R, Iran Polym J., 2010, 19(8), 635-646. 